\title{
Natural history of periocular capillary haemangiomas: changes in internal blood velocity and lesion volume
} lesion volume

\section{Abstract}

Aim Although the clinical characteristics of childhood periocular capillary haemangiomas are well known, serial measurements of blood velocity and lesion size are unknown. This investigation was designed to measure the changes in maximum blood velocity and estimated size of lesion in children with capillary haemangioma not requiring active intervention.

Study design Retrospective case-note review for a cohort of children with capillary haemangioma involving the eyelid and orbit. Patients and methods Children with periocular capillary haemangioma, under the care of the Orbital unit at Moorfields Eye Hospital between 1996 and 2005, were monitored clinically and with repeated ultrasonographic examination. Volume estimates were calculated as an ovoid based on the three maximum orthogonal measurements for the haemangioma, and blood velocity was assessed by Colour Flow Mapping, Colour Doppler Energy Imaging, and Spectral Doppler techniques using a Sequoia 512 Acuson scanner.

Results Twenty-four children (12 boys) had initial assessment by 18 months of age, and the haemangioma increased in size in 14/24 (58\%), the increase being between 4 and $931 \%$ of initial volume estimate. The largest measured size for an individual haemangioma appears inversely related to the child's age at measurement, this mirroring a similar trend in measurements for the maximum blood velocity. Blood velocity measurements also tend to decrease with time, the peak velocity being before 1 year of age in the majority (15/24; 62\%). In many children, both volume estimates and blood velocities show a cyclic variation - this occurring with increasing intervals between the maxima, before a final decay in both parameters.

Although, for the whole group, there was no correlation (correlation coefficient $=0.29$ )

between estimated size and measured blood velocity, some individual children showed a significant correlation between the two parameters. The age at maximum blood velocity appeared to precede the age at maximum volume in most children, and in many there was an orbital anomaly detectable on ultrasonographic examination, even with complete clinical resolution of the haemangioma.

Conclusions Ultrasonographic examination of periocular capillary haemangiomas show that these lesions have a very high blood velocity in feeding vessels - about 2-3 orders of magnitude greater than normal capillary beds - and that the velocity and volume of such lesions undergo a cyclic variation during their natural history. Evidence suggests that both velocity and volume decrease with time, although often not returning to zero on ultrasonography (unlike the clinical resolution of the lesions). In most children, blood velocity peaks before volume estimates and this might suggest that decreasing perfusion leads to later tissue atrophy and involution of the haemangioma.

Eye (2006) 20, 1228-1237. doi:10.1038/sj.eye.6702387

Keywords: capillary haemangioma; orbit; Doppler ultrasonography; blood velocity

Introduction

Capillary haemangioma is the commonest childhood orbital vascular anomaly, ${ }^{1,2}$ typically 
presenting within a few months of birth as bluish swelling of the eyelid or anterior orbital mass, and generally enlarges during infancy with a later gradual involution over several years. Most haemangiomas pose no threat to visual development, but larger lesions cause amblyopia owing to mechanical ptosis or astigmatism. Their origin and the processes that underlie their behaviour are unknown, although there is circumstantial evidence linking them with tissue of placental origin. ${ }^{3}$

Doppler ultrasonography is the noninvasive investigation of choice in the diagnosis of most vascular lesions, ${ }^{4,5}$ and characteristic findings for capillary haemangiomas include acoustic heterogeneity, a high density of vessels throughout the lesions, and a very high internal blood flow velocity - the latter being unusual in other childhood orbital tumours, such as rhabdomyosarcoma. Linear measurement of an orbital mass in three orthogonal dimensions allows an estimation of the volume. ${ }^{6}$ The spectrum of velocity within orbital capillary haemangiomas and the changes of velocity during tumour growth and involution are, however, unreported - as are the temporal changes in dimensions of such lesions.

We report a series of children with orbital capillary haemangioma, in whom serial Doppler ultrasonography measurements of blood velocity and lesion size were acquired. The changes in the natural history for these two parameters were examined in relation to each other, and also in relation to clinical changes in the orbital lesions.

\section{Materials and methods}

The clinical case notes were reviewed for children who, between 1996 and 2005, had been under the care of one surgeon (GER) for capillary haemangiomas involving the eyelid and orbit. Apart from routine ophthalmic clinical examination, at most visits, the child underwent orthoptic assessment and also examination by a highly experienced ophthalmic ultrasonographer (MR). Ethical committee review was not required for this retrospective review of routine clinical practice.

\section{Ultrasonographic methods}

\section{Size of haemangiomas}

Children were seated, often on their parent's knees, and examined using a Sequoia 512-Acuson (Siemens) ultrasound scanner. The orbit was examined through closed eyelids using a linear array probe (centre frequency $10 \mathrm{MHz}$ ) smeared with a coupling gel and the extent of the orbital mass initially assessed using real-time B-mode (grey-scale) imaging. As accurate estimation of an irregular three-dimensional lesion is difficult with a single cross-sectional image, the haemangiomas were measured in two orthogonal B-mode planar sections, and the transverse, longitudinal, and maximum anterior-to-posterior dimensions were noted (Figure 1a) and repeated at follow-up visits.

Transverse and sagittal sections were taken at $1 \mathrm{~mm}$ intervals through the lesion and the two orthogonal sections displaying the greatest antero-posterior dimensions were selected: the dimensions recorded were transverse base (' $x$ '), longitudinal base $\left({ }^{\prime} y\right.$ '), and antero-posterior distance ('depth'; ' $z$ '). The size of the haemangioma was estimated by assuming a rotational ellipsoid, as described previously, ${ }^{6}$ in which the volume is calculated by the formula:

$$
\text { Estimated Volume }=\frac{4 \pi(x \cdot y \cdot z)}{3}
$$

\section{Blood flow within haemangiomas}

Blood flow studies included Colour Flow Mapping (CFM), Colour Doppler Energy (CDE) imaging, and Spectral Doppler techniques.

CFM is a technique whereby blood flow is imaged, in colour, in conjunction with the static structure displayed on a conventional grey-scale B-mode image. Echo displacement is converted into velocity measurements within a selected area of the B-mode image, the detected 'line-of-sight' velocity being displayed in shades of red (flow towards the probe) and blue (flow away from the probe). CFM in capillary haemangiomas tends to demonstrate a major blood vessel, either in red or blue, with variations in hue and brightness; in contrast, the image from vascular tumours tends to be a mosaic of blues and reds, owing to the varying directions of flow within multiple internal vessels. Although a gate may be set on the velocity range, an 'aliasing' artefact may occur if the gate is too low - this being seen as colour 'wraparound', with higher velocity flows towards the probe being wrongly identified in blue. An intentionally low-velocity gate may, however, increase the sensitivity in detecting very slow blood flow at the edge of vessels. Where the aliased image is hard to interpret (Figure $1 \mathrm{~b}$ and $\mathrm{d}$ ), the examiner may elect to determine spatial density of blood vessels using CDE technique.

$\mathrm{CDE}$ is of particular use when information regarding blood flow, but without directional information, is required. The technique, based on similar physical principles to CFM, uses the intensity of a single colour (red) to indicate the local volume of blood flow; conventionally, a red scale is used with intensity being approximately proportional to the number of moving cells. The image does not actually show speed of flow. Although its inability to show flow speed and direction 

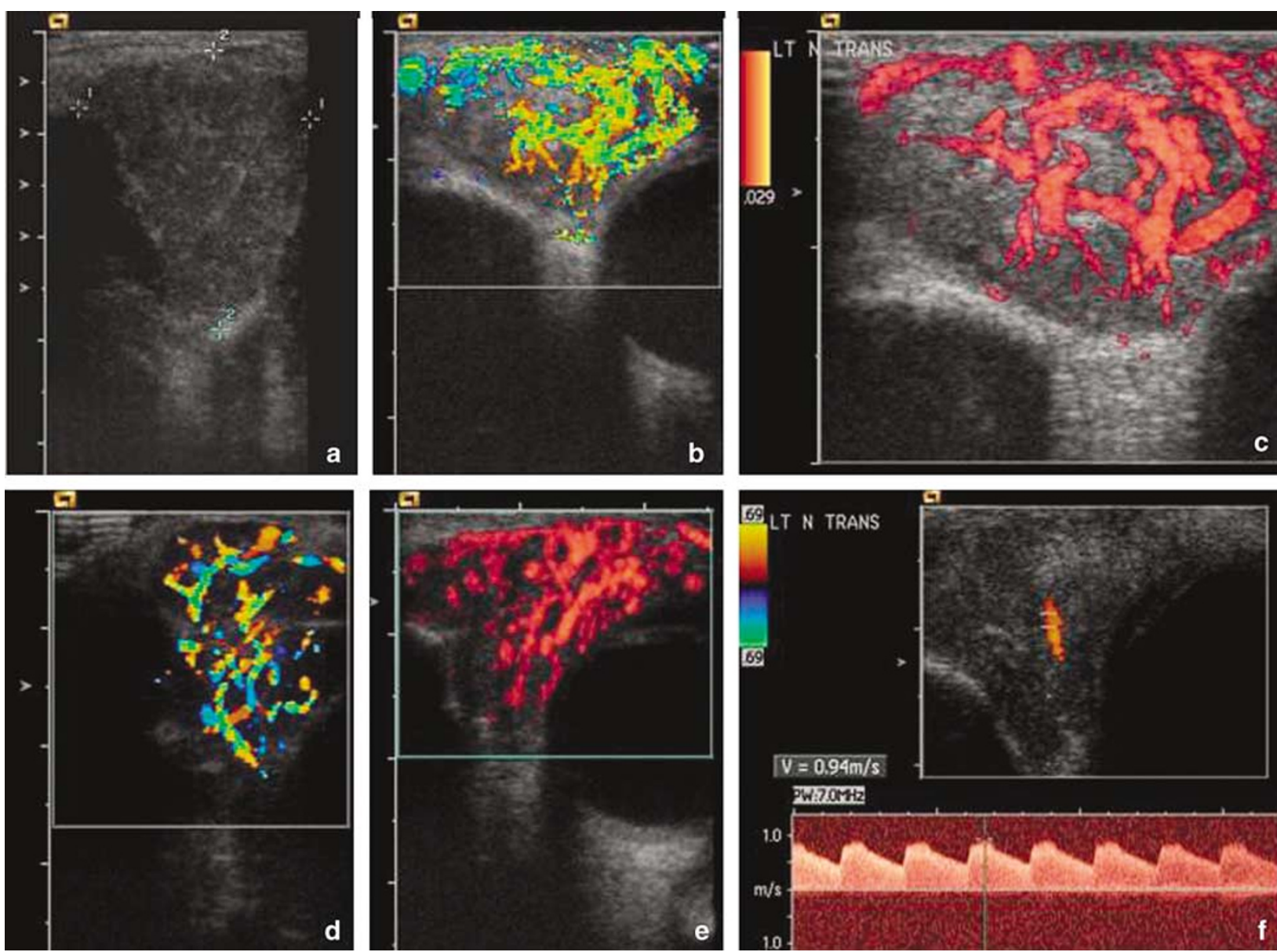

Figure 1 Doppler ultrasonography methods to investigate capillary haemangioma. (a) Horizontal plane B-mode section of haemangioma with electronic calipers positioned to measure transverse base (caliper 1: $22.5 \mathrm{~mm}$ ) and antero-posterior depth (caliper 2: $27.1 \mathrm{~mm}$ ). (b) Colour Flow Mapping (CFM) displaying 'aliased' image, with low-velocity scale set (velocity range +3 to $-3 \mathrm{~cm} / \mathrm{s}$ ). (c) Colour Doppler Echo image of same haemangioma as (b), with low-velocity scale set (velocity range +3 to $-3 \mathrm{~cm} / \mathrm{s}$ ). (d) CFM displaying 'aliased' image, with low-velocity scale set (velocity range +6 to $-6 \mathrm{~cm} / \mathrm{s}$ ). (e) Colour Doppler Echo image of same haemangioma as (d), with low-velocity scale set (velocity range +2 to $-2 \mathrm{~cm} / \mathrm{s}$ ). (f) CFM of same haemangioma as (d) and (e), with high-velocity scale set (velocity range +69 to $-69 \mathrm{~cm} / \mathrm{s}$ ) - only one blood vessel being imaged now. The two horizontal white lines on the vessel indicate the chosen setting for the spectrogram gate, the underlying spectrogram showing a maximum systolic velocity of $94 \mathrm{~cm} / \mathrm{s}$.

are disadvantages, $\mathrm{CDE}$ is particularly useful for imaging capillary haemangiomas as it does not exhibit 'aliasing', and so the velocity scale can be set very low to increase the sensitivity to flow. This technique is used to estimate either the spatial density (fraction of selected area taken up by red) or number density of blood vessels (number of vessels per unit area; arbitrarily, $\geq 5$ vessels $/ \mathrm{cm}^{2}$ in capillary haemangioma).

CFM is undertaken after determining the spatial density of vessels using $\mathrm{CDE}$, the velocity scale on the CFM being increased from a very low setting - such as 2 or $3 \mathrm{~cm} / \mathrm{s}$ (where 'aliasing' will be evident; Figure 1c) - to a high velocity scale (such as $70 \mathrm{~cm} / \mathrm{s}$ ), where only one or two high-velocity blood vessels will be detected within the lesion (Figure 1c and e). This sequence facilitates selection of vessels with the highest velocity, for measurement of maximal systolic velocity within the tumour - this velocity being measured using a Spectral Doppler Technique.

Spectral Doppler provides a graphical output, known as a Spectrogram, comprising a time-related display of the blood flow velocity at a particular vascular location; the location 'gate' is selected by the ultrasonographer and is displayed as two horizontal white lines on the image (Figure 1f). As vessels are small in capillary

haemangiomas, the 'gate' usually encompasses the entire vascular diameter and the spectrogram shows the entire range of velocities present in the gated region-ranging from slow flow near the vessel wall, to high velocity centrally within the vessel (Figure 1f). Flow towards the 
probe is conventionally displayed above the $X$-axis and viceversa.

When first examined, the nature of the orbital lesion was confirmed by its acoustic heterogeneity, by a vascular density of greater than $5 / \mathrm{cm}^{2}$, and by highvelocity systolic flow in at least one vessel. At this - and subsequent-visits, the three orthogonal dimensions were measured, the highest recorded systolic velocity noted, and control measurements taken from the corresponding position in the contralateral unaffected orbit. On the rare occasions when a child was uncooperative, comment was made in the notes and no measurements recorded.

\section{Data presentation}

To facilitate comparison of the two parameters, velocity and size measurements are each presented, as a proportion of the maximum recorded, on the same ordinand and the nonparametric data were plotted as a continuous curve using a Microsoft Excel ${ }^{\circledR}$ spreadsheet.

Statistical methods included $\chi^{2}$ analysis, Fisher's exact test where an expected cell value was less than 5 , Spearman's test to determine correlation between velocity and size, and unpaired Student's $t$-test to compare parametric data.

\section{Results}

Twenty-four infants (12 boys) with unilateral orbital capillary haemangiomas were reviewed in the Orbital Clinic on several occasions between 1996 and 2005; children requiring medical intervention for amblyopia were not included.

The lesion affected seven right orbits (29\%), the lower eyelid in 10/24 (42\%) cases, the upper lid is 13/24 (54\%) and the medial canthus in one child. A total of 166 data sets were collected, with a mean of 6.9 attendances per child (range 1-17). The average age at which the parents became aware of the lesion was 2.1 months (range 0-14) (Table 1), the mean age at first clinic visit was 9.4 months (range 3-48), and the last recorded visit was 44.4 months (range 10-100) - the average clinical follow-up being 35 months (range 23-91).

The maximum computed size ranged from 0.92 to $8.98 \mathrm{ml}$ (mean 4.16, median $3.86 \mathrm{ml}$ ) and the age for this maximum volume was between 2 and 60 months (mean 7.2, standard deviation 15.7; median 14 months). The maximum-recorded blood velocity ranged from 15 to $91 \mathrm{~cm} / \mathrm{s}$ (mean 52, standard deviation 22.9; median $45.5 \mathrm{~cm} / \mathrm{s}$ ) (Table 2); the ages for these maximum velocities varying widely, between 3 and 52 months (mean 14.2, standard deviation 13.3; median 8.5 months) (Figure 2).
Table 1 Clinical and ultrasonographic characteristics of 24 children with periocular capillary haemangioma

\begin{tabular}{lccc}
\hline Characteristic & Mean & $\begin{array}{c}\text { Standard } \\
\text { deviation }\end{array}$ & Range \\
\hline Age (months) at & & & \\
$\quad$ First symptom & 2.1 & 2.9 & $0-14$ \\
$\quad$ First assessment & 9.4 & 9.9 & $3-48$ \\
$\quad$ Last assessment & 44.4 & 25 & $10-100$ \\
Number of assessments per child & 6.9 & 4.4 & $1-17$ \\
Greatest blood velocity (cm/s) & 52 & 22.5 & $15-91$ \\
Greatest estimated volume (ml) & 4.13 & 1.66 & $0.92-8.98$ \\
\hline
\end{tabular}

\section{Volume and velocity profiles}

There was a wide variation in the highest recorded volumes and velocity for haemangiomas (Figure 2), and it would appear that the maximum velocity is not related to the maximum estimated size. There were several patterns of decay over time (Figure 3). In none was there the expected exponential decay of either parameter but, instead, there were significant oscillations where follow-up data were sufficient.

\section{Calculated volume profiles}

In all but four cases, the volume increased after first measurement: of the 17 infants first assessed at under 1 year of age, $13(76 \%)$ had an increase in size - of between 4 and $456 \%$ (mean increase 102\%). All 24 had initial assessment by the age of 18 months, and 14/24 $(58 \%)$ haemangiomas showed a highly variable increase in size - by up to 9 times the initial volume (4-931\%). Albeit recognising that the true largest volume for the haemangioma might be missed between clinic measurements, there appears to be an inverse relationship between the maximum estimated volume and the age at measurement (Figure 4).

Fourteen patients had five or more data sets: 10/14 $(71 \%)$ showed more than one oscillation in volume profile and 7/14 had 3-4 oscillations before apparent involution of the haemangioma (Figure 3). In the 14 children showing such oscillations, the interval between peaks becomes longer - the mean interval between first and second peaks being 8 months, whereas that between the second and third peaks was 18 months $(P<0.001)$; the mean interval between the third and fourth peaks for children 1 and 3 was 15.5 months.

Twenty-two children had three or more volume estimates and in only $6 / 22(27 \%)$ was the final volume $10 \%$ (or less) of the greatest estimate during the follow-up period. Despite clinical resolution of the haemangioma, there was persistence of an ultrasonographically-detectable lesion in the $4 / 8$ 
Table 2 Number of ultrasonographic assessments - together with the maximum recorded velocity, greatest estimated volume, and relationship between the two-during the follow-up interval for 24 children with periocular capillary haemangioma

\begin{tabular}{|c|c|c|c|c|c|c|}
\hline Child number & $\begin{array}{l}\text { Number of } \\
\text { assessments }\end{array}$ & $\begin{array}{l}\text { Follow-up } \\
\text { interval } \\
\text { (months) }\end{array}$ & $\begin{array}{c}\text { Maximum } \\
\text { velocity }(\mathrm{cm} / \mathrm{s})\end{array}$ & $\begin{array}{l}\text { Maximum } \\
\text { size }(\mu l)\end{array}$ & $\begin{array}{l}\text { Correlation } \\
\text { coefficient }^{\text {a }}\end{array}$ & $\begin{array}{l}\text { Velocity/volume } \\
\text { profile }\end{array}$ \\
\hline 1 & 17 & 43 & 72 & 7230 & 0.92 & Oscillating \\
\hline 2 & 16 & 92 & 33 & 3148 & 0.58 & Nonspecific \\
\hline 3 & 13 & 41 & 85 & 3002 & 0.34 & Oscillating \\
\hline 4 & 10 & 70 & 27 & 4480 & 0.31 & Nonspecific \\
\hline 5 & 10 & 57 & 40 & 4911 & 0.54 & Oscillating \\
\hline 6 & 10 & 45 & 25 & 2469 & -0.35 & Oscillating \\
\hline 7 & 9 & 41 & 91 & 2111 & -0.69 & Nonspecific \\
\hline 8 & 9 & 67 & 35 & 1981 & 0.71 & Oscillating \\
\hline 9 & 9 & 40 & 63 & 4116 & 0.59 & Oscillating \\
\hline 10 & 9 & 41 & 52 & 6240 & -0.69 & Nonspecific \\
\hline 11 & 8 & 51 & 47 & 3992 & -0.01 & Oscillating \\
\hline 12 & 6 & 41 & 35 & 3726 & 0.62 & Oscillating \\
\hline 13 & 5 & 38 & 82 & 7316 & -0.01 & Oscillating \\
\hline 14 & 4 & 31 & 60 & 4415 & 0.73 & Oscillating \\
\hline 15 & 4 & 10 & 48 & 6162 & 0.8 & $<5$ data points \\
\hline 16 & 4 & 11 & 90 & 3344 & 0.9 & $<5$ data points \\
\hline 17 & 4 & 17 & 44 & 4563 & -0.81 & $<5$ data points \\
\hline 18 & 3 & 38 & 78 & 3383 & 0.99 & $<5$ data points \\
\hline 19 & 3 & 9 & 36 & 918 & 0.99 & $<5$ data points \\
\hline 20 & 3 & 6 & 80 & 3407 & 0.96 & $<5$ data points \\
\hline 21 & 3 & 28 & 41 & 2055 & -0.19 & $<5$ data points \\
\hline 22 & 3 & 13 & 25 & 4365 & 0.50 & $<5$ data points \\
\hline 23 & 2 & 4 & 15 & 2720 & - & $<5$ data points \\
\hline 24 & 1 & 5 & 43 & 8975 & - & $<5$ data points \\
\hline
\end{tabular}

${ }^{\mathrm{a} C}$ Correlation coefficient between volume and flow measurements within the series for each child.

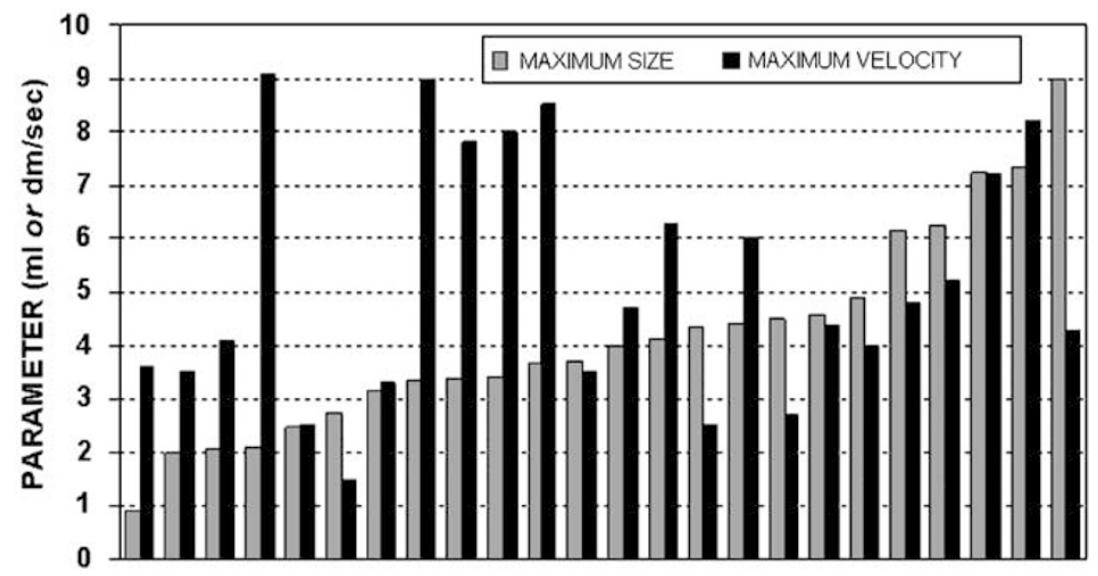

Figure 2 Maximum estimated size $(\mathrm{ml})$ and peak velocity $(\mathrm{dm} / \mathrm{s})$ in 24 children with periocular capillary haemangiomas not requiring active intervention; ranked by increasing maximum size.

children followed beyond 4 years of age; the lesion being undetectable in the other four children on assessments at $50,53,88$, and 100 months. In eight (33\%) children, half followed to beyond their third birthday, the last volume estimate was similar to, or higher than, the initial measurement.

\section{Velocity profiles}

Peak velocity measurements within haemangiomas show an overall decline with age (Figure 5), although the highest velocity for each individual child was variable and poorly related to age (Figure 6). Velocity 
CHILD NUMBER 1

Maxima: Velocity $72 \mathrm{~cm} / \mathrm{sec}$; Volume $7.2 \mathrm{ml}$

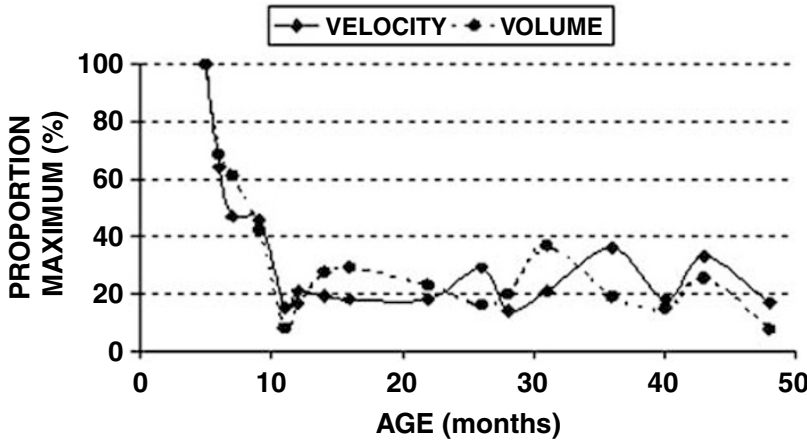

CHILD NUMBER 8

Maxima: Velocity $35 \mathrm{~cm} / \mathrm{sec}$; Volume $2.0 \mathrm{ml}$

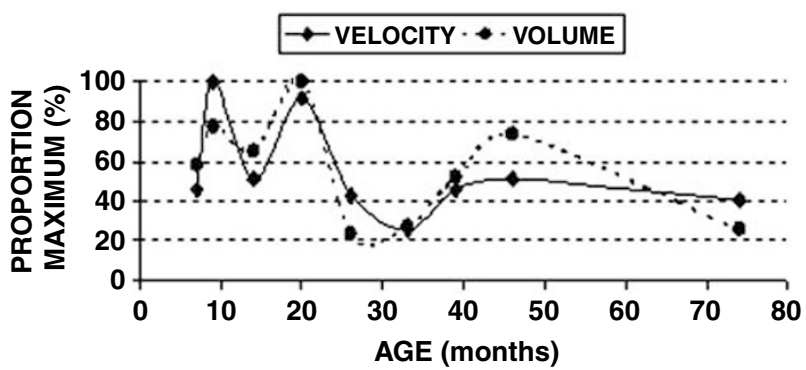

CHILD NUMBER 3

Maxima: Velocity $85 \mathrm{~cm} / \mathrm{sec}$; Volume $3.7 \mathrm{~m}$

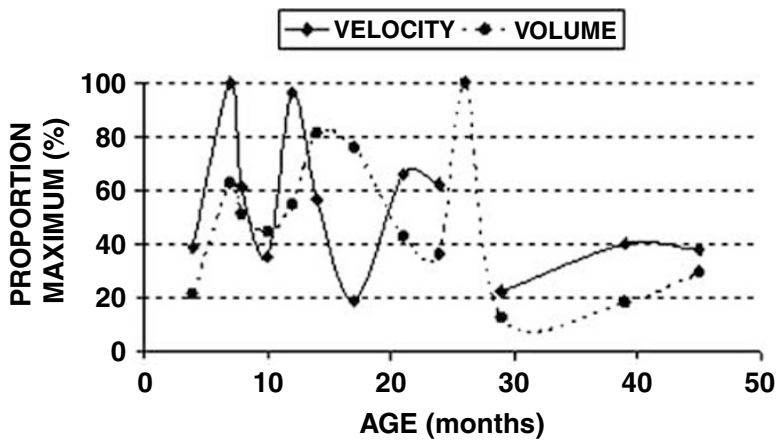

CHILD NUMBER 7

Maxima: Velocity $91 \mathrm{~cm} / \mathrm{sec}$; Volume $2.1 \mathrm{ml}$

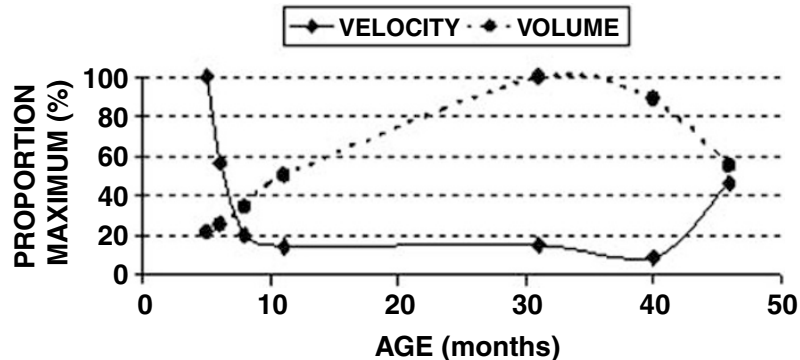

Figure 3 Volume and velocity profiles, compared by plotting as a proportion (\%) of the maximum value during follow-up: four children showing either a damped oscillating pattern for volume and velocity (children 1,3, and 8) or as an atypical pattern (child number 7).

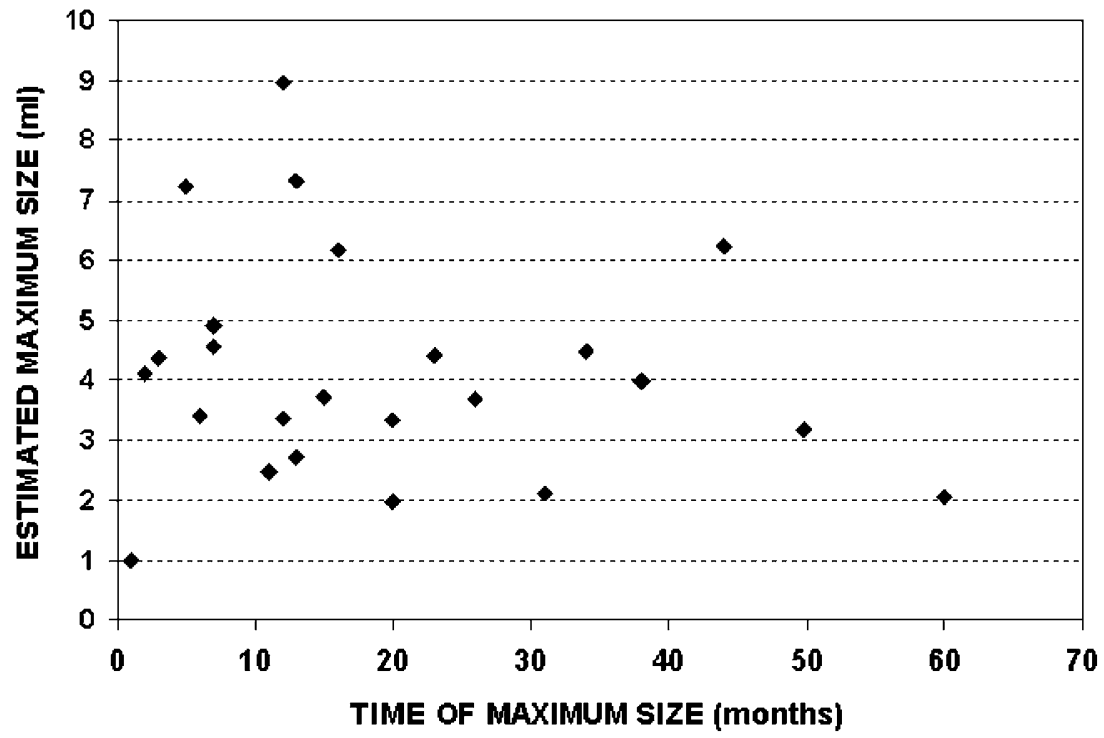

Figure 4 Maximum estimated size in relation to the age at which this was measured.

measurements increased after initial assessment in 10/23 $(43 \%)$ children and in $15 / 23(65 \%)$, the peak velocity was before the age of 1 year. In 17 children assessed within a year of birth, the velocity increased in nine (53\%) before 12 months of age - with an average rise of $83 \%$
(15-160\%). In contrast to the volume profiles, further rises in velocity were rare after 1 year of age.

Of the 13 children with five or more velocity measurements, $10(77 \%)$ showed more than one oscillation of the profile and seven (54\%) showed more 


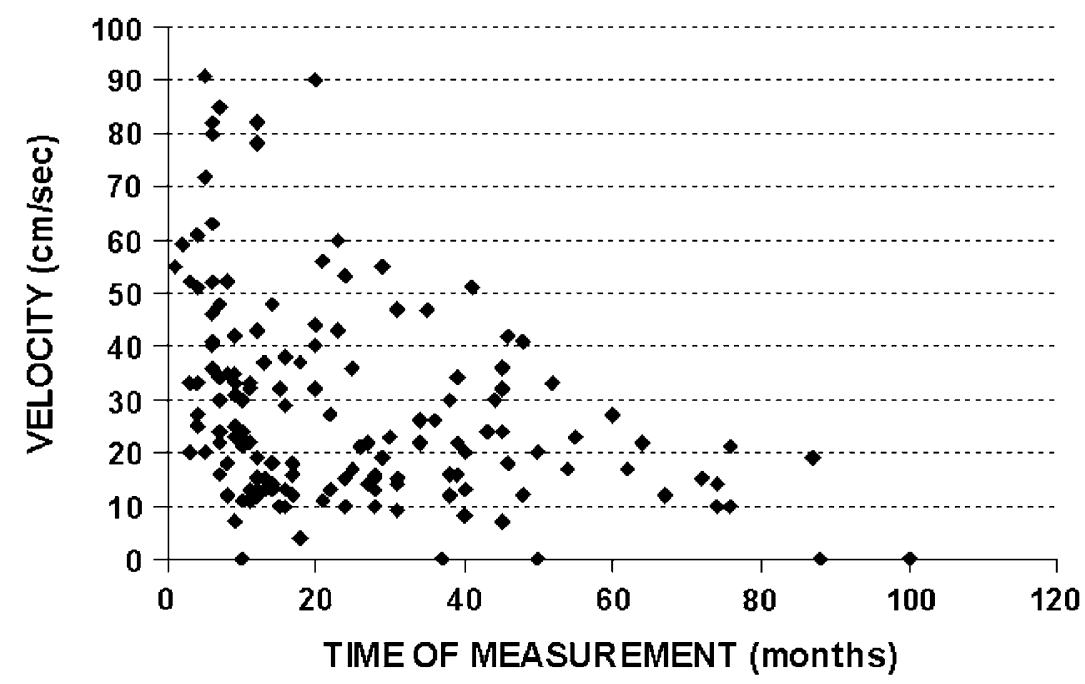

Figure 5 Velocity measurements within capillary haemangiomas: complete data for all children, showing a poor correlation with age, but a clear trend for very high velocity only in infants and decreasing velocity at older ages.

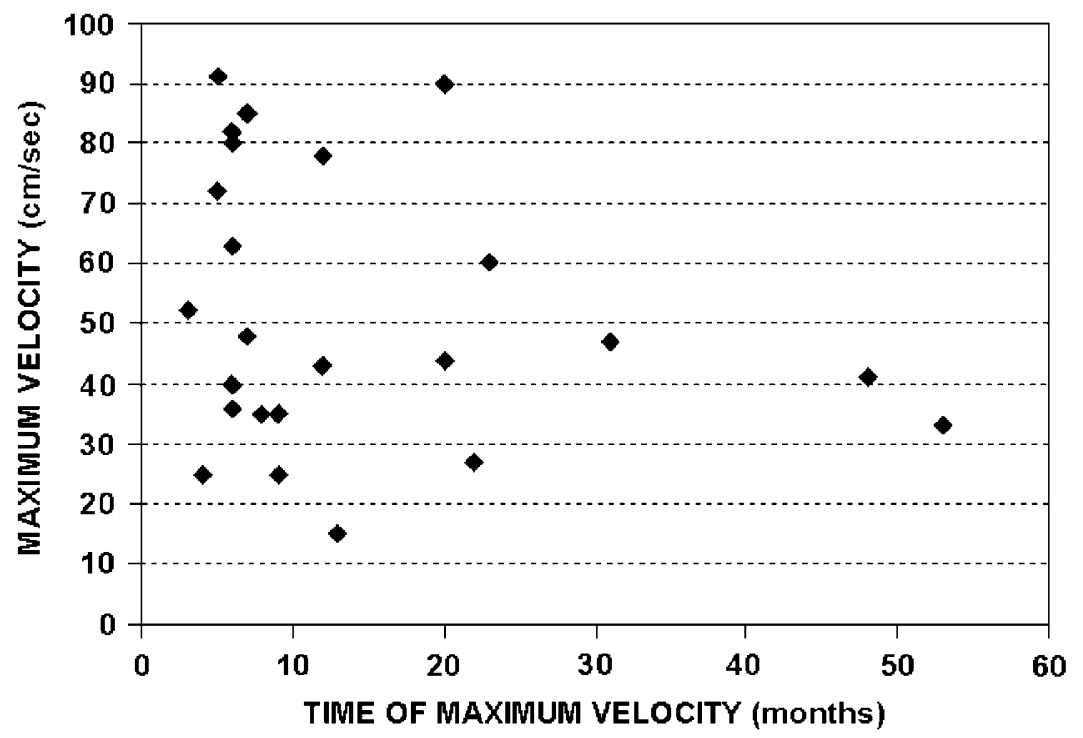

Figure 6 Maximum velocity for each child, related to the age at which this was measured.

than two oscillations; more than two or three oscillations were exceptional. As with volume oscillations, the interval between the first and second peaks in these seven children was significantly shorter (mean 5.4 months) than that between the second and third peaks (mean 13.9 months) $(P<0.01)$; where a third interval occurred - in three children - this was marginally longer (mean 16.3 months; $P>0.05$ ).

Final velocity measurements decayed to undetectable levels in only $3 / 22(14 \%)$ children - this occurring at 37, 50 , and 88 months - and most $(9 / 22 ; 41 \%)$ children continued to have abnormal vessels detectable ultrasonographically late in the course of the condition.

\section{Relationship between volume and velocity}

The relationship between estimated volume and its corresponding velocity is poor for the whole group (Figure 7; correlation coefficient $=0.29$ ), but some individuals shows a significant correlation between the two measurements (Table 2) (Figure 8). A comparison of age at maximum volume and that for highest measured flow (Figure 9) suggests that, in most haemangiomas, the flow peaks before the volume $(11 / 22$ children; compared to $3 / 22$ with the reverse); this might suggest that a decreasing vascular perfusion might result in a later tissue atrophy and regression of the haemangioma. 
The behaviour of haemangiomas appears to fall into one of the two main types. The first group, of 10 children, shows a 'classic' profile with both velocity and volume oscillating with decreasing amplitude and prolonged interval before decaying to a small, clinically undetectable, lesion with minimum perfusion (Figure 3); the volume and velocity measurements appearing to show considerable correlation (Figure 8). A variant profile is apparent in a second, smaller, group of five patients, in whom both measurements exhibit a more prolonged fluctuation before decay (Figure 3), this being for as long as 80 months in some cases (children 2 and 4).

For nine patients, there were insufficient data sets to ascribe a particular pattern of decay, although a close association between the velocity/volume data sets was observed in three of them.

\section{Discussion}

The clinical behaviour of capillary haemangiomas is well described, including the presentation and growth in infancy, with subsequent involution. ${ }^{1,2}$ Although Doppler ultrasonography is widely used in the diagnosis of vascular lesions, there have not been any reports with serial measurements of periorbital haemangiomas. ${ }^{4,5}$ This study was designed to define more accurately the natural history of these lesions, and to identify whether volume and or velocity measurements have clinical prognostic value.

Although the examinations over a 10 -year period (average 6.9 per child, with follow-up 35 months) were performed by the same, highly experienced ultrasonographer (MR), there are limitations to the ultrasonographic methods. First, size variations may

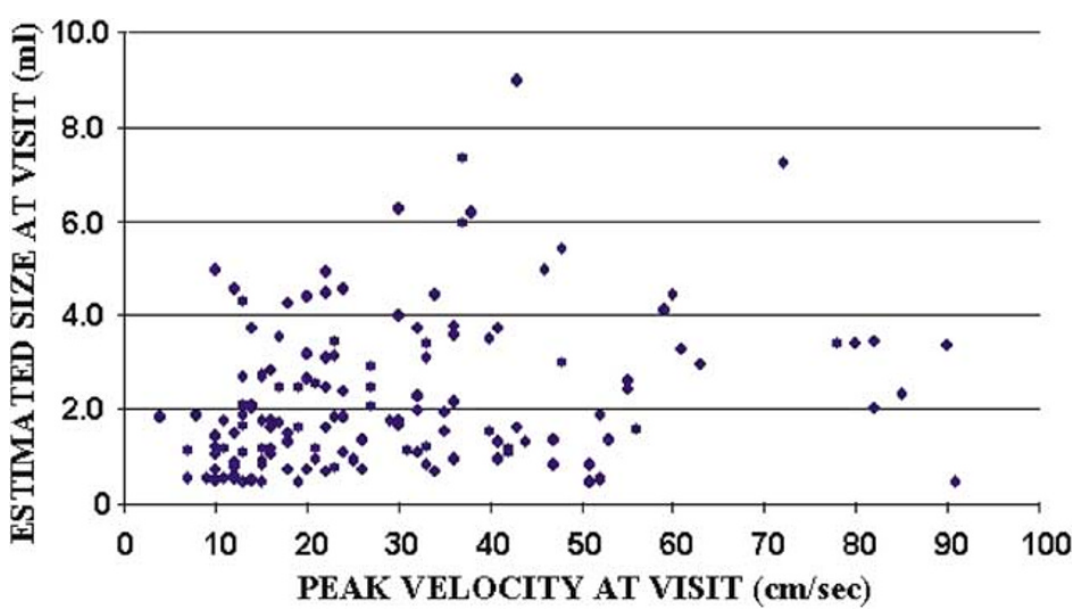

Figure 7 Relation between measured velocity and the estimated volume for capillary haemangioma; the complete data set shows a poor overall correlation (correlation coefficient $=0.29$ ).

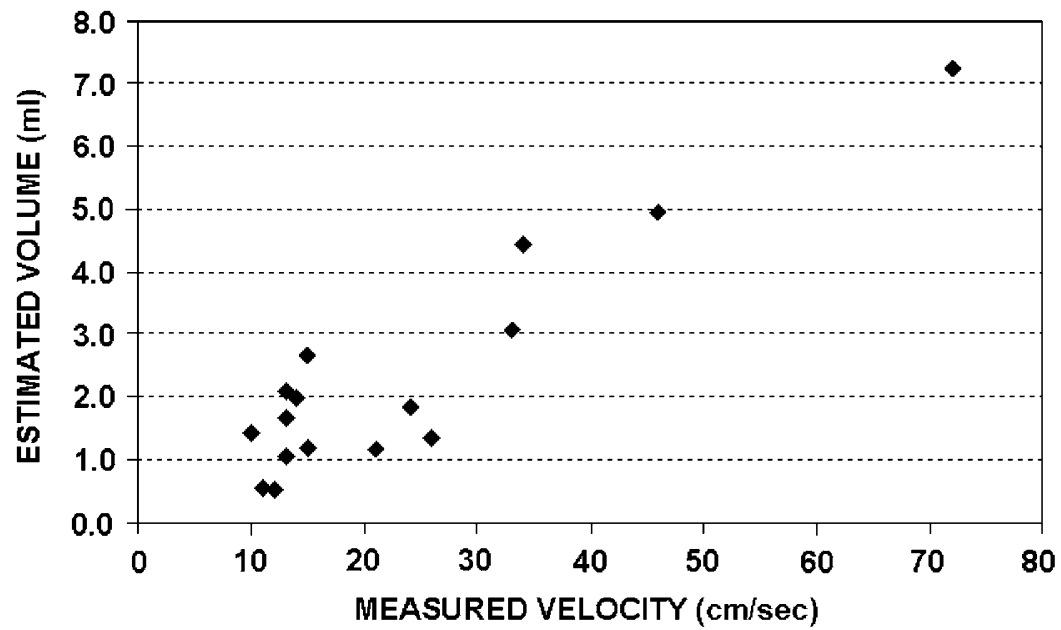

Figure 8 For some children, as in child number 1 illustrated, there was a close correlation between estimated volume and velocity within the haemangioma (correlation coefficient $=0.92$ ). 


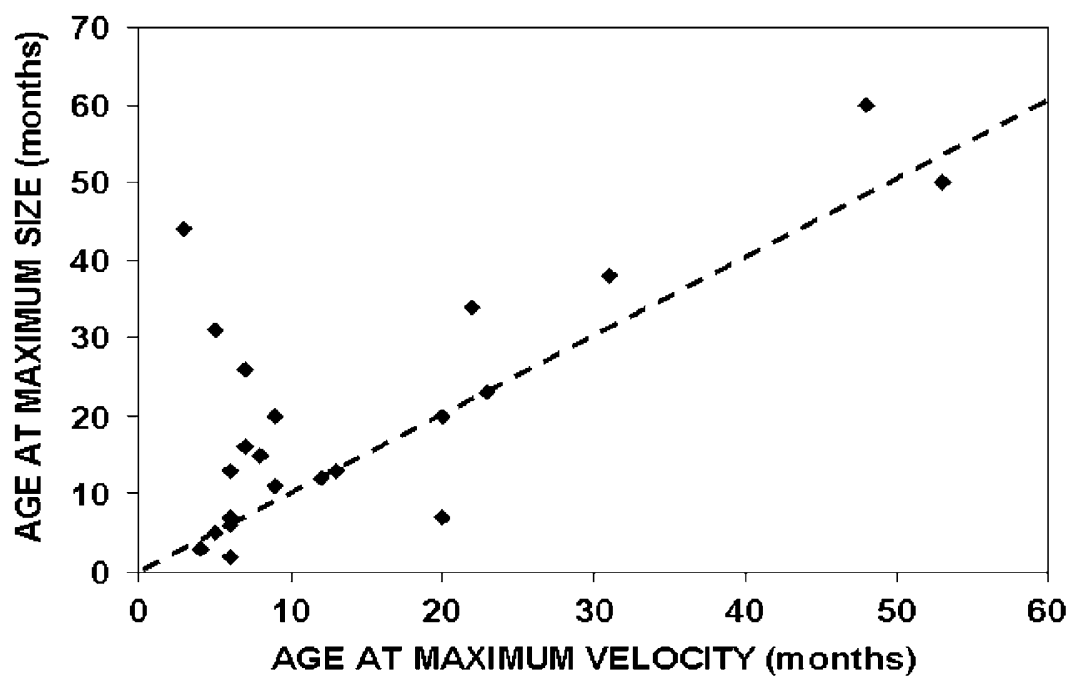

Figure 9 Age at maximum volume compared with the age at maximum velocity, for each individual haemangioma in 22 children with two or more assessments of the parameters. Most haemangiomas fall above the line of equality (dashed line), suggesting that the peak blood velocity most commonly occurs several months before the occurrence of maximum volume.

be due to vascular engorgement, rather than true tissue hypertrophy or proliferation; it would, however, be difficult to attribute the large changes in size (up to a nine-fold increase) solely to vascular engorgement. Secondly, although flow rates are measured, this does not necessarily reflect volume flow, as there is no measure for the cross-sectional area of the vessel. Thirdly, it is possible that flow rates and volume might have been affected by the emotional state of the child, although it was possible to get most measurements without great difficulty and they were not recorded where the child was fractious; it is unlikely that the observed large changes in flow and volume were solely attributable to short-term fluctuations in the haemangiomas.

A very high velocity (up to about $90 \mathrm{~cm} / \mathrm{s}$ ) has been demonstrated within the major vessels in capillary haemangiomas, especially in infancy - this contrasting with typical flows of $50 \mathrm{~cm} / \mathrm{s}$ in adult aorta and about $0.1 \mathrm{~cm} / \mathrm{s}$ in a normal capillary bed. As the feeder vessels show flow rates up to three orders of magnitude higher than that in a true capillary bed, this would suggest that capillary haemangiomas are not physiologically normal capillaries: it being likely that they represent capillarybased arteriovenous shunts, possibly fed directly from a major orbital artery. The observed oscillations in size and flow would suggest that capillary haemangiomas comprise a network of vessels, through different parts of which perfusion varies over weeks to months. Vascular proliferation and involution might also vary within different parts of the lesion, this also affecting local perfusion and flow rates - the degree of perfusion possibly being influenced by local growth factors, vascular tone, and apoptosis. As - most commonly - the highest flow rates appear to precede the maximum volume, this might suggest that decreasing tissue perfusion heralds tissue atrophy; the frequent persistence of an ultrasonographically detectable anomaly, despite complete clinical resolution, also suggests that vascular perfusion slowly decreases with loss of tissue bulk - this leaving a connective tissue residuum with a few larger vessels. Allowing for oscillations in volume and flow, the overall trend appears to follow that of an exponential decay and the volume 'spikes' may reflect either limited growth, or areas of enhanced perfusion, superimposed upon this background of involution.

Some children show a fair correlation between lesion volume and velocity, although one parameter was not predictive for the other. It might be postulated that increased perfusion, with higher velocity, might lead to both congestive enlargement of the haemangioma and also increased capillary endothelial hypertrophy; the corollary would be that the increased metabolic requirements of proliferating endothelial tumour would necessitate increased flow through the main feeder vessels. The similarity of profiles between some children, and the concordance of short-term repeated measures in individual lesions, suggests that these changes are largely due to changes in tissue mass - rather than fluctuation in minute-to-minute perfusion.

Capillary haemangiomas may be of placental origin and their growth and atrophy, or possibly apoptosis, may be controlled by maternal vascular growth factors that have crossed the placenta. ${ }^{3}$ The typical early growth of these lesions, confirmed in our study with both volume and velocity measurements, may represent a postpartum release from maternal growth factors. The influence of 
maternal chemokines should, however, be short-lived and other local growth factors might be implicated in the cycles of lesion enlargement and involution that have been observed in most children within this study.

From a clinical perspective, orbital capillary haemangiomas enlarge (most doubling) in over threequarters of children under the age of 1 year. With prolonged follow-up, a similar proportion of children show two or more oscillations in volume, each 'spike' occurring at increasing intervals; as most haemangiomas undergo complete clinical resolution, these apparent growth spikes should not engender anxiety in the clinician or parents if the visual development remains unaffected.

\section{References}

1 Haik BG, Jakobiec FA, Ellsworth RM, Jones IS. Capillary haemangioma of the lids and orbit: an analysis of the clinical features and therapeutic results in 101 cases. Ophthalmology 1979; 86: 760-792.

2 Haik BG, Karcioglu ZA, Gordon RA, Pechous BP. Capillary Haemangioma (Infantile periocular haemangioma). Surv Ophthalmol 1994; 38: 399-426.

3 North PE, Waner M, Mizeracki A, Mrak RE. A unique microvascular phenotype shared by juvenile hemangiomas and human placenta. Arch Dermatol 2001; 137: 559-570.

4 Sleep TJ, Fairhurst JJ, Manners RM, Hodgkins PR. Doppler ultrasonography to aid diagnosis of orbital capillary haemangioma in neonates. Eye 2002; 16: 316-319.

5 Neudorfer M, Leibovitch I, Stolovitch C, Dray JP, Hermush V, Nagar $\mathrm{H}$, Kessler A. Intraorbital and periorbital tumors in children - value of ultrasound and color Doppler imaging in the differential diagnosis. Am J Ophthalmol 2004; 137: 1065-1072.

6 Richtig E, Langmann G, Mullner K, Richtig G, Smolle J. Calculated tumour volume as a prognostic parameter for survival in choroidal melanomas. Eye 2004; 18: $619-623$ 